\title{
Traumatismes Balistiques en Zone Opérationnelle: Expérience de l'Hôpital Niveau 2 Togo de Kidal
}

\author{
Biréga Koutora, \\ Kossigan Ayi Amavi, \\ Yao Messanvi Akpoto, \\ Damessane Lamboni, \\ Gnimdou Akala-Yoba, \\ Mibirim Agbogawo, \\ Mahèza Akpanahe,
}

Clinique Médico-Chirurgicale (Pavillon Militaire), CHU Sylvanus Olympio, Lomé, Togo

Badjona Songne-Gnamkoulamba,

Service des Urgences Chirurgicales, CHU Sylvanus Olympio, Lomé

Faculté des Sciences de la Santé, Université de Lomé, Togo

\section{Résumé}

Introduction : Les traumatismes balistiques sont à l'origine de lésions pluritissulaires variables dont la prise en charge est différente de la traumatologie civile. Le but de cette étude est de rapporter l'expérience de l'Hôpital Niveau 2 Togo de Kidal sur les traumatismes balistiques en zone opérationnelle au Nord-Mali. Méthodologie: Il s'est agi d'une étude rétrospective descriptive et transversale sur 22 mois couvrant la période du $1^{\text {er }}$ Novembre 2015 au 31 Août 2017 ; incluant les blessés par projectiles admis à l'Hôpital Niveau 2 Togo de Kidal. Résultats : Cent cinquante-et-deux blessés dont 149 hommes et 3 femmes ont été enregistrés. L'âge médian était de 29,6 ans (extrêmes : 19 ans et 56 ans). Les militaires étaient les plus atteints (92,8\%). Les lésions étaient provoquées par les éclats d'obus dans $73,7 \%$ des cas. Elles étaient dominées par les lésions des tissus mous $(65,1 \%)$, suivies des fractures $(15,1 \%)$. Les membres étaient les sites les plus touchés. La prise en charge consistait à la recherche et à la correction des détresses vitales, et à la prise en charge spécifique des lésions balistiques. L'évolution était favorable chez 115 blessés $(75,6 \%)$. Vingt-et-un blessés $(13,8 \%)$ avaient été évacués vers un hôpital de niveau supérieur. Il y avait 16 cas (10,5\%) de décès dont 15 $(9,8 \%)$ avant l'admission. Conclusion : La prise en charge des blessés selon les principes de la chirurgie de guerre conditionne le pronostic. 
Mots clés : Traumatisme balistique, Zone opérationnelle, Nord-Mali

\title{
Ballistic Trauma in Operational Zone: Experience of Togo Level 2 Hospital of Kidal
}

\author{
Biréga Koutora, \\ Kossigan Ayi Amavi, \\ Yao Messanvi Akpoto, \\ Damessane Lamboni, \\ Gnimdou Akala-Yoba, \\ Mibirim Agbogawo, \\ Mahèza Akpanahe,
} Clinique Médico-Chirurgicale (Pavillon Militaire), CHU Sylvanus Olympio, Lomé, Togo Badjona Songne-Gnamkoulamba, Service des Urgences Chirurgicales, CHU Sylvanus Olympio, Lomé Faculté des Sciences de la Santé, Université de Lomé, Togo

\begin{abstract}
Introduction: Ballistic trauma are responsible for variable multitissue lesions which management is different from the usual traumatology. The purpose of this study is to report the experience of Togo Level 2 Hospital of Kidal on ballistic trauma in operational zone in North Mali. Methodology : It was a retrospective descriptive and transversal study on 22 months, from November 1, 2015 to August 31, 2017 ; including projectiles injured admitted to the Togo Level 2 Hospital of Kidal. Results : One hundred and fifty-two injured including 149 men and 3 women were registered. The average age was 29.6 years (extremes : 19 years and 56 years). The soldiers were the most affected $(92.8 \%)$. Shrapnel were the main lesion agents $(73.7 \%)$. Soft tissue lesions were predominant (65.1\%), followed by fractures (15.1\%). Members were the most affected sites. The management consisted of the search and the correction of the vital distress, and the specific care of ballistic lesions. The outcome was favorable for 115 injured (75.6\%). 21 injured (13.8\%) were evacuated to a higher level hospital. 16 cases (10.5\%) of death including 15 $(9.8 \%)$ before admission were registered. Conclusion : The management of injured according to the priciples of war surgery conditions the prognosis.
\end{abstract}


Keywords : Ballistic trauma, Operational zone, North-Mali

\section{Introduction}

Le traumatisme balistique est la conséquence du transfert d'énergie d'un projectile sur l'organisme. Ce projectile peut être une balle tirée d'une arme à feu, ou un éclat provenant de l'enveloppe ou du contenu d'un engin explosif: grenade, roquette, obus, bombe d'aéronefs, mine et bombe artisanales ou engins explosifs improvisés (Club des Anesthésistes Réanimateurs et Urgentistes Militaires, 2008 ; Rouvier, 1997). Les traumatismes balistiques surviennent dans les conflits armés, mais aussi lorsque les armes sont utilisées en temps de paix (Giannou, 2009). Rares en pratique civile, ces traumatismes sont fréquents dans les conflits armés actuels à l'instar du conflit malien. Les lésions engendrées sont variables et diversifiées, et leur prise en charge répond à des règles spécifiques, différentes de la traumatologie civile. Le respect des principes de la chirurgie de guerre dans la prise en charge des blessés balistiques sur un théâtre d'opérations est un facteur de meilleur pronostic (Club des Anesthésistes Réanimateurs et Urgentistes Militaires, 2008 ; Giannou, 2009). Cette étude a été initiée afin de rapporter l'expérience de l'Hôpital Niveau 2 Togo (HN2 Togo) de Kidal, sur les traumatismes balistiques au Nord - Mali, dans les opérations engagées par l'Organisation des Nations Unies (ONU), dans le cadre de la Mission multidimensionnelle Intégrée des Nations Unies pour la Stabilisation au Mali (MINUSMA).

\section{Patients et méthode d'étude}

Il s'est agi d'une étude rétrospective descriptive et transversale couvrant la période du $1^{\text {er }}$ Novembre 2015 au 31 Août 2017 (22 mois). Etaient inclus dans l'étude, les blessés par projectiles (balles ou éclats d'engins explosifs) admis à l'HN2 Togo de Kidal.

A l'admission, les blessés étaient pris en charge de façon standardisée, avec une prise en charge des détresses vitales suivie d'un examen clinique conduit de la tête aux pieds. Le bilan paraclinique comportait systématiquement une radiographie standard chez tous les blessés admis vivants, et au besoin un bilan biologique fait de : groupe sanguin-facteur Rhésus, numération formule sanguine, urémie, créatininémie et glycémie. Les lésions étaient classées suivant la gravité et le type de structures lésées selon la classification Croix-Rouge des blessures de guerre (Tableau I). Le traitement médicamenteux comportait systématiquement une antibioprophylaxie à base de Ceftriaxone, la séro-anatoxinothérapie antitétanique, des antalgiques associés ou non à des anti-inflammatoires non stéroïdiens, et d'autres médicaments selon le besoin. Tous les patients admis 
vivants avaient bénéficié d'un traitement chirurgical fait de parage des plaies avec extraction possible des projectiles, et suture immédiate ou primaire différée.

Les paramètres étudiés étaient : l'âge, le sexe, le pays d'origine, le statut dans la MINUSMA, les circonstances de survenue, le bilan lésionnel, les aspects thérapeutiques et le pronostic à court terme.

Le traitement des données a été fait à partir du tableur Excel 2013. Les images ont été traitées sur le logiciel Paint.

Tableau I : Classification Croix-Rouge des blessures de guerre (Giannou, 2009)

\begin{tabular}{|c|c|c|}
\hline \multicolumn{2}{|c|}{ Classification } & Description \\
\hline \multirow{3}{*}{$\begin{array}{l}\text { Degré de } \\
\text { gravité }\end{array}$} & Degré 1 & $\begin{array}{c}\text { Orifice d'entrée + Orifice de sortie } \leq 10 \mathrm{~cm} \text {; la cavité de la blessure } \\
\text { ne peut admettre } 2 \text { doigts avant le parage ; et absence de fracture ou } \\
\text { présence de fracture simple. }\end{array}$ \\
\hline & Degré 2 & $\begin{array}{l}\text { Orifice d'entrée }+ \text { Orifice de sortie } \leq 10 \mathrm{~cm} \text {; la cavité de la blessure } \\
\text { peut admettre } 2 \text { doigts avant le parage ; ou fracture communitive. }\end{array}$ \\
\hline & Degré 3 & $\begin{array}{l}\text { Orifice d'entrée }+ \text { Orifice de sortie } \geq 10 \mathrm{~cm} \text {; la cavité de la blessure } \\
\text { peut admettre } 2 \text { doigts avant le parage ; ou fracture communitive }\end{array}$ \\
\hline \multirow{4}{*}{$\begin{array}{l}\text { Types de } \\
\text { lésions }\end{array}$} & Type TM & $\begin{array}{l}\text { Lésions des tissus mous ; Absence de fracture ; Absence de lésions } \\
\text { de structures vitales. }\end{array}$ \\
\hline & Type F & $\begin{array}{l}\text { Fractures simples ou communitives ; Absence de lésions de } \\
\text { structures vitales }\end{array}$ \\
\hline & Type V & $\begin{array}{c}\text { Lésions de structures vitales mettant en jeu la vie du patient } \\
\text { (Système nerveux central, Thorax, Abdomen, Vaisseaux); Absence } \\
\text { de fracture. }\end{array}$ \\
\hline & Type VF & $\begin{array}{l}\text { Blessures avec fractures et touchant les structures vitales, mettant en } \\
\text { jeu la vie du patient, ou risquant d'exiger une amputation. }\end{array}$ \\
\hline \multirow{3}{*}{$\begin{array}{l}\text { Corps } \\
\text { étrangers à la } \\
\text { radiographie }\end{array}$} & M0 & Absence de corps étranger \\
\hline & M1 & Un corps étranger visible \\
\hline & M2 & Plusieurs corps étrangers visibles \\
\hline
\end{tabular}

\section{Résultats}

\section{Aspects épidémiologiques}

En 22 mois, 152 cas de traumatismes balistiques ont été enregistrés, soit une fréquence mensuelle de 6,9 cas. L'âge médian des blessés était de 29,6 ans (extrêmes : 19 ans et 56 ans). Il s'agissait de 149 hommes et de 3 femmes, soit une sex-ratio de 49,7. La tranche d'âge de 25 ans à 35 ans était la plus touchée $(59,9 \%)$. Selon le pays d'origine, les patients originaires de la Guinée étaient majoritaires (50\%), suivis de ceux du Tchad (36,2\%). Il s'agissait majoritairement des militaires de l'ONU (92,8\%). Les civils de l'ONU étaient atteints dans $2,6 \%$ des cas. Le tableau II récapitule les paramètres épidémiologiques. 
Tableau II : Récapitulatif des paramètres épidémiologiques des traumatismes balistiques en zone opérationnelle : Hôpital Niveau 2 Togo de Kidal

\begin{tabular}{cccc}
\hline & & Effectif & $\%$ \\
\hline Sexe & Masculin & 149 & 98,0 \\
& & 3 & 2,0 \\
Tranche d'âge & {$[19-25[$} & 27 & 17,8 \\
& {$[25-35[$} & 91 & 59,9 \\
& {$[35-45[$} & 23 & 15,1 \\
Statut MINUSMA & {$[45-55[$} & 9 & 5,9 \\
& $\geq 55$ & 2 & 1,3 \\
& Militaire & & \\
Pays d'origine & Civil & 141 & 92,8 \\
& Personnel non UN & 4 & 2,6 \\
& Personnel de police & 3 & 2,6 \\
& Guinée & & 2,0 \\
& Tchad & 76 & 50,0 \\
& Mali & 55 & 36,2 \\
& Bangladesh & 7 & 4,6 \\
& Népal & 6 & 3,9 \\
& Tunisie & 4 & 2,6 \\
& Burkina Faso & 2 & 1,3 \\
& & 1 & 0,7 \\
& & 1 & 0,7 \\
\hline
\end{tabular}

\section{Aspects lésionnels}

Les lésions étaient provoquées par les éclats d'obus dans la majorité des cas $(73,7 \%)$. Les autres agents lésionnels étaient les balles issues de tirs tendus d'armes à feu $(17,8 \%)$, et les éclats de mines et engins explosifs improvisés $(8,5 \%)$.

Selon la classification Croix-Rouge des blessures de guerre, on notait : suivant le degré de gravité, une prédominance des lésions de degré 2 (98 cas / $64,5 \%)$; et selon le type de structures lésées, une prédominance des lésions de type TM (99 cas / 65,1\%) suivie des lésions de type F (23 cas / 15,1\%). Les lésions de type $\mathrm{V}$ étaient notées dans 12 cas (7,9\%). Selon le nombre de corps étrangers métalliques visibles à la radiographie, on avait : aucun corps étranger dans 12 cas $(7,9 \%)$; un seul corps étranger dans 91 cas $(59,8 \%)$; et plusieurs corps étrangers dans 33 cas $(21,7 \%)$. Le nombre de corps étrangers n'avait pas été évalué chez 16 blessés $(10,5 \%)$ car décédés avant leur admission ou pendant la prise en charge. Les figures 1,2 et 3 montrent des exemples respectivement de lésions de type TM, de type $\mathrm{F}$ et de type $\mathrm{V}$. 
Figure 1: Exemple de lésion de type TM. Plaie balistique du bras gauche par éclat d'obus. $\mathrm{A}=$ orifice d'entrée ; $\mathrm{B}=$ image du projectile à la radiographie.
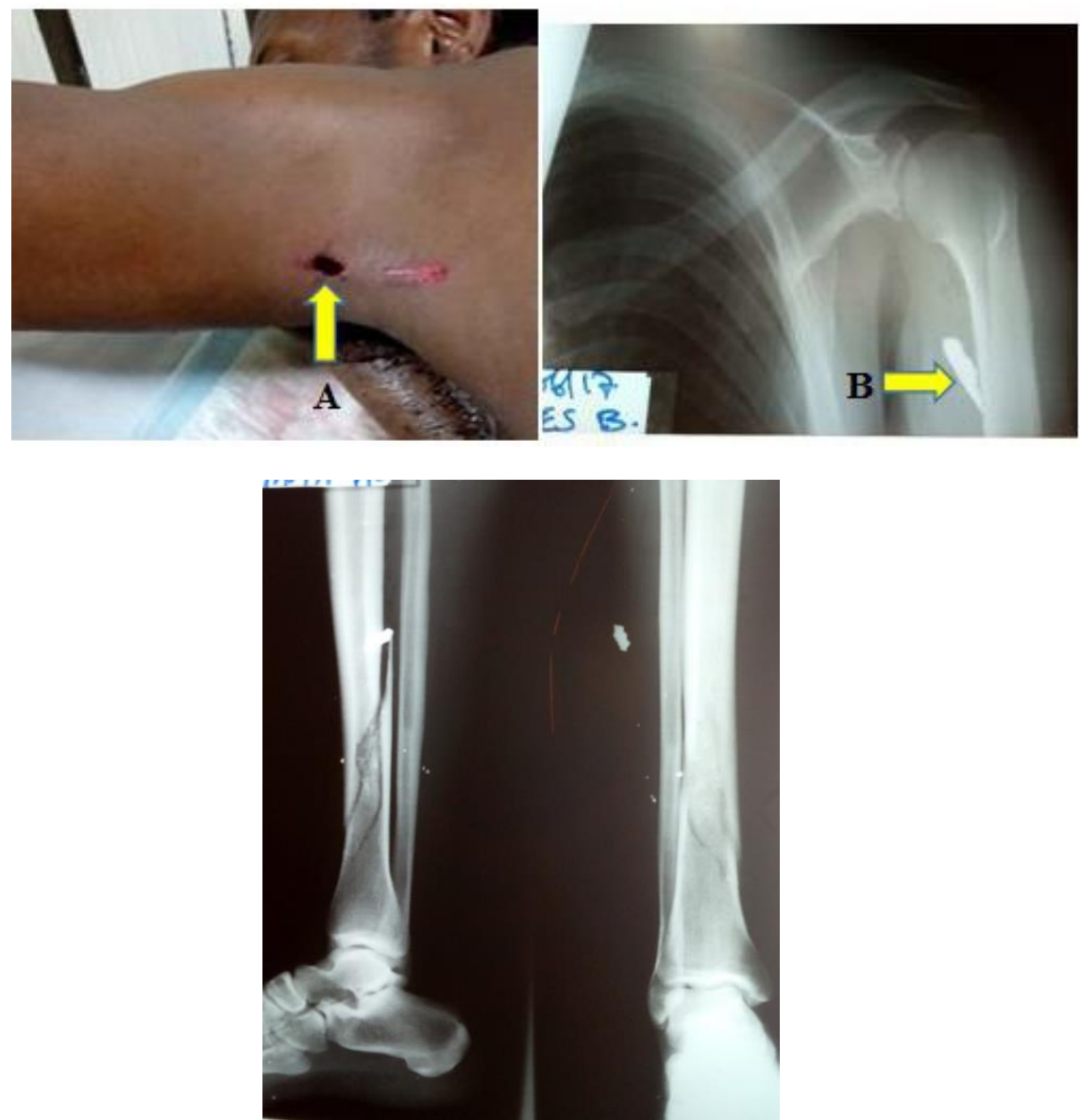

Figure 2: Exemple de lésion de balistique de jambe par éclats d'engin explosif improvisé.type F. Fracture

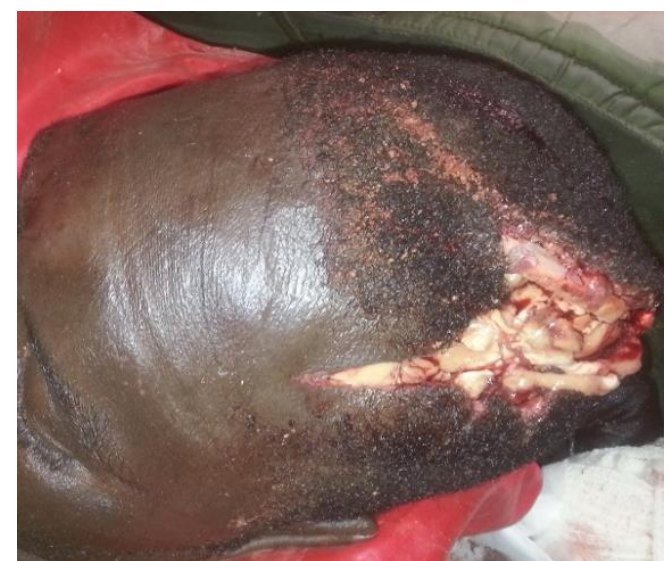

Figure 3: Exemple de lésion de type V. Plaie crânio-cérébrale par balle. 
La topographie des lésions était unique dans 99 cas $(65,1 \%)$. On notait des associations topographiques dans 53 cas (34,9\%). Les membres thoraciques étaient les plus atteints $(38,1 \%)$, suivis des membres pelviens (33,5\%). Le thorax était touché chez $21,0 \%$ des blessés et l'abdomen chez $12,5 \%$. Le tableau III récapitule le bilan lésionnel des traumatismes balistiques enregistrés à l'Hôpital Niveau 2 Togo de Kidal.

Tableau III: Récapitulatif du bilan lésionnel des traumatismes balistiques en zone opérationnelle : Hôpital Niveau 2 Togo de Kidal

\begin{tabular}{|c|c|c|c|}
\hline & & Effectif & $\%$ \\
\hline \multirow[t]{3}{*}{ Agents lésionnels } & Eclats d'obus & 112 & 73,7 \\
\hline & Balles & 27 & 17,8 \\
\hline & Eclats de mines et EEI* & 13 & 8,5 \\
\hline \multirow[t]{3}{*}{ Gravité des lésions } & Degré 1 & 11 & 7,2 \\
\hline & Degré 2 & 98 & 64,5 \\
\hline & Degré 3 & 43 & 28,3 \\
\hline \multirow[t]{4}{*}{ Types de lésions } & Type TM & 99 & 65,1 \\
\hline & Type F & 23 & 15,1 \\
\hline & Type V & 12 & 7,9 \\
\hline & Type VF & 18 & 11,9 \\
\hline \multirow[t]{6}{*}{ Topographie } & Membre thoracique & 58 & 38,1 \\
\hline & Membre pelvien & 51 & 33,5 \\
\hline & Tête & 37 & 24,3 \\
\hline & Thorax & 32 & 21,0 \\
\hline & Abdomen & 19 & 12,5 \\
\hline & Cou & 6 & 3,9 \\
\hline
\end{tabular}

\section{Aspects thérapeutiques}

La prise en charge consistait au prime abord chez tous les blessés à la recherche et à la correction des détresses vitales. Dix-sept patients $(11,2 \%)$ avaient reçu une transfusion sanguine. Tous les patients admis vivants avaient bénéficié d'un traitement chirurgical fait de parage des plaies avec extraction possible des projectiles, et suture immédiate ou primaire différée. La suture après parage était immédiate pour les plaies du cuir chevelu et de la face, et primaire différée pour les plaies des autres parties du corps. Le délai médian pour la suture primaire différée était de 3,1 jours (extrêmes : 2 jours et 5 jours). Les figures 4 et 5 montrent respectivement des images : de projectile extrait d'une plaie balistique de la paroi thoracique ; et d'une plaie balistique gingivo- 
vestibulo-labiale inférieure parée et suturée immédiatement. L'immobilisation des membres s'est faite à l'aide d'attelles, ou de plâtres circulaires fenêtrés. Une ostéosynthèse par fixateur externe avait été faite chez 6 blessés $(3,9 \%)$. Une laparotomie avait été faite chez 4 blessés, et une cervicotomie chez 2 blessés.
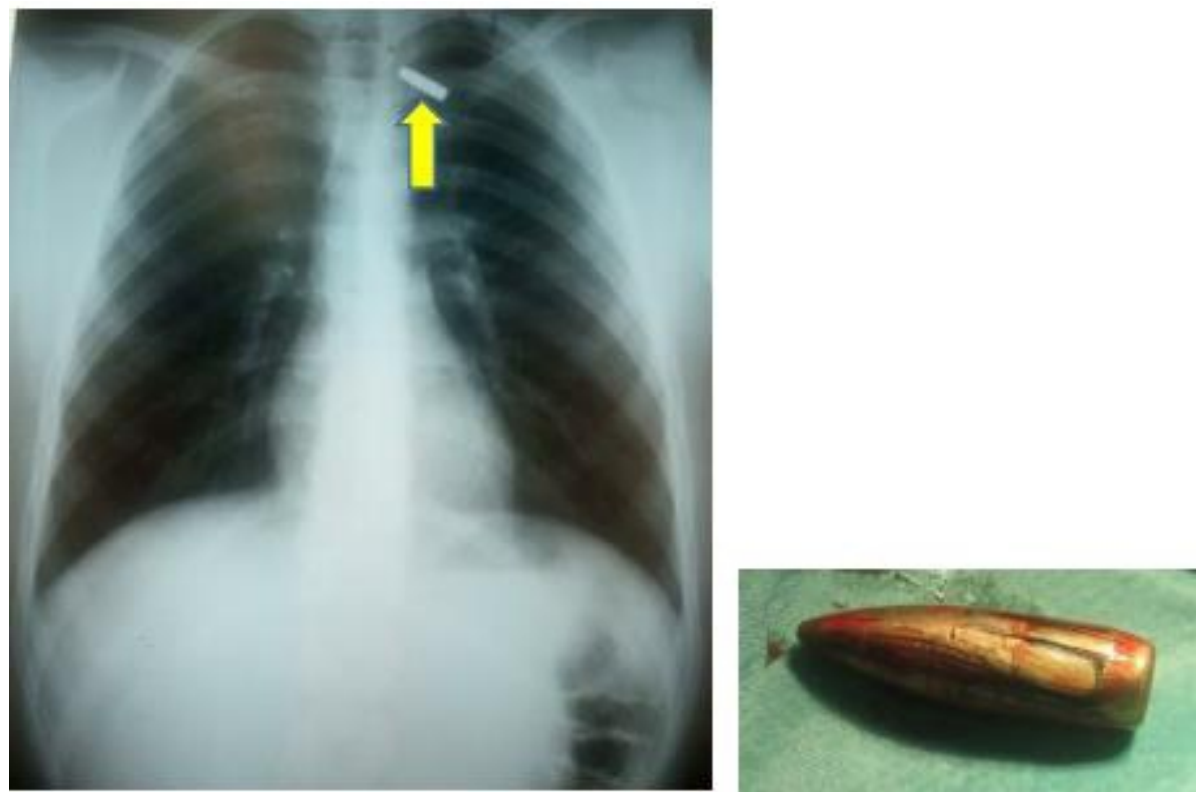

Figure 4: Projectile (balle) extrait d'une plaie balistique de la paroi thoracique

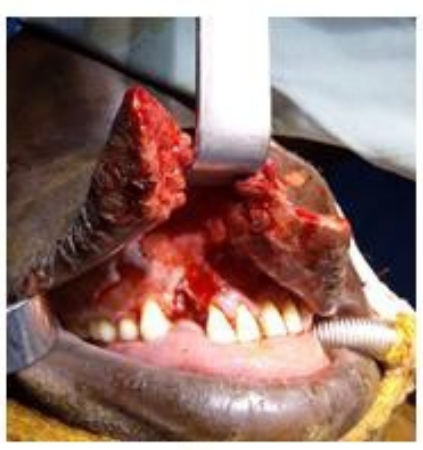

A

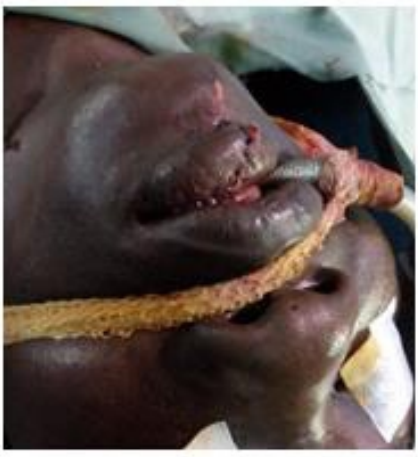

B

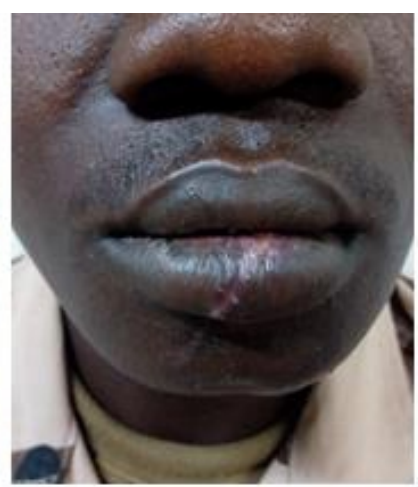

C

Figure 5: Plaie balistique gingivo-vestibulo-labiale inférieure parée et suturée immédiatement. $\mathrm{A}=$ Aspect de la plaie après le parage $; \mathrm{A}=$ Aspect après la suture $; \mathrm{C}=$ Aspect à la cicatrisation.

\section{Aspects évolutifs}

$\mathrm{Au}$ plan pronostique, on avait enregistré 16 décès (10,5\%) dont 15 $(9,8 \%)$ avant leur admission à l'hôpital et $1(0,7 \%)$ pendant la prise en charge. Vingt-et-un blessés $(13,8 \%)$ avaient été évacués vers un hôpital de niveau 
supérieur après avoir été stabilisés à l'HN2 Togo de Kidal. Cent quinze blessés (75,6\%) avaient eu une évolution à court terme favorable et avaient été libérés à partir de l'HN2 Togo. Le délai médian de séjour hospitalier était de 3 jours (extrêmes : 0 et 16 jours).

\section{Discussion}

Les traumatismes balistiques sont fréquents dans le conflit malien. Les attaques par obus, mines et engins explosifs improvisés constituent les principaux moyens responsables de ces lésions. Il s'agit de moyens tactiques de plus en plus utilisés dans les conflits armés actuels dont le caractère est non conventionnel et asymétrique (Donat, 2009 ; Haus-Cheymol, 2011). Le jeune âge de la majorité des patients de cette série et la prédominance masculine concordent avec la force déployée. Les militaires des unités combattantes de la force sont les plus touchés en raison de la mission qui leur est assignée.

La physiopathologie des traumatismes balistiques repose sur le comportement du projectile dans l'organisme, comportement dépendant des facteurs balistiques et anatomiques (Rouvier, 1997 ; Giannou, 2009 ; Fabeck, 2017). Les facteurs balistiques sont : l'énergie du projectile, la distance de la cible, la nature et les propriétés du projectile (Club des Anesthésistes Réanimateurs et Urgentistes Militaires, 2008 ; Giannou, 2009 ; Haouam, 2018). En effet, un traumatisme balistique correspond à un transfert d'énergie entre un projectile et l'organisme. Ce transfert est fonction de l'énergie initiale du projectile, proportionnelle à la moitié de sa masse et au carré de sa vitesse $\left(\mathrm{E}=1 / 2 \mathrm{mv}^{2}\right)$. Ainsi, les projectiles à hautes vitesses ont donc théoriquement le pouvoir vulnérant le plus important. Aussi, plus la distance de la cible est petite, plus graves sont les lésions engendrées. Enfin, indépendamment de la vitesse du projectile, le transfert d'énergie va dépendre de la nature du projectile (balle, éclats), de sa capacité à s'écraser ou à se fragmenter, et de sa stabilité (effet de bascule, de rotation). Tous ces éléments en augmentant la surface de transfert d'énergie à l'organisme vont aggraver les lésions observées. Quant aux facteurs anatomiques, il s'agit de la densité et de l'élasticité des tissus concernés par le traumatisme (Rouvier, 1997). Plus la densité est élevée et l'élasticité faible, plus important sera le transfert d'énergie. Ainsi, les structures osseuses sont celles à haut transfert d'énergie, avec pour conséquence la possibilité de fractures complexes. Les organes à haute teneur en eau, denses et peu élastiques comme les organes pleins abdominaux, le cœur, le cerveau, sont le siège de lésions à type d'éclatement et de broiement. Les organes à haute teneur en air, élastiques et peu denses comme le poumon sont résistants au traumatisme balistique du fait du faible transfert d'énergie en l'absence de fragmentation du projectile. En pratique, aucune théorie physique ne permet cependant de prévoir avec certitude le comportement d'un projectile dans le corps humain (Rouvier, 1997). Le 
clinicien ne sait pas toujours quelle arme a été à l'origine de la blessure, et il ne connaît jamais l'énergie cinétique disponible au point d'impact. De ce fait, seule l'étendue des lésions tissulaires permet de juger de l'importance du transfert d'énergie (Giannou, 2009). Toutefois, la connaissance des notions de balistique est importante pour l'amélioration de la prise en charge des blessures par projectiles, et le développement des moyens de protection (Club des Anesthésistes Réanimateurs et Urgentistes Militaires, 2008 ; Giannou, 2009).

La répartition lésionnelle ne suit pas une distribution aléatoire mais est la résultante des effets de la visée ennemie, des effets de protection balistique et du type d'arme (Donat, 2009). Dans cette étude, les membres thoraciques étaient les plus atteints suivis des membres pelviens et de la tête. Il en a été de même dans d'autres études (Donat, 2009 ; Saint-Julien, 2013). L'évolution des moyens de protection du combattant (blindage des véhicules, port de casques et de gilets pare-balles) entraîne une modification des sites lésionnels classiques, comme la réduction relative des plaies thoraco-abdominales en faveur des plaies du segment céphalique et des membres (Donat, 2009 ; SaintJulien, 2013).

Sur le plan thérapeutique, la prise en charge du traumatisé balistique sur un théâtre d'opérations vise avant tout la recherche et la correction des détresses vitales selon le principe de Damage Control Resuscitation (Giannou, 2009 ; Chauvin, 2002). Ce qui permet de réduire la mortalité dite évitable. La réduction de la mortalité évitable passe par : le contrôle et la compensation des hémorragies, la sécurisation des voies aériennes, et la prévention de l'hypothermie par couverture isolante (Saint-Julien, 2013 ; Daban, 2012). Une fois stabilisés, les blessés sont évacués selon le besoin vers un hôpital d'infrastructure où la prise en charge définitive est assurée. La prise en charge des plaies balistiques est différente de celle des plaies qui ne sont pas d'origine projectilaire. En effet la suture des plaies balistiques est souvent différée à cause de l'importance des dégâts des parties molles et de la forte contamination microbienne. Ainsi, ce principe de non fermeture immédiate des plaies balistiques est fondamental, car il réduit le risque septique en raison du caractère potentiellement contaminé de la plaie même après le parage le plus soigneux (Giannou, 2009 ; Chauvin, 2002). Il existe cependant des cas particuliers notamment les plaies balistiques du cuir chevelu, de la face et les plaies articulaires. Les immobilisations par attelles, par plâtres fenêtrés et les exo-fixations en cas de fractures associées sont des moyens favorisant la cicatrisation (Chauvin, 2002 ; Bruner, 2011 ; Khechimi, 2015).

$\mathrm{Au}$ plan pronostique, la mortalité par traumatisme balistique enregistrée à l'HN2 Togo de Kidal était de 10,5\% dont 9,8\% avant l'admission à l'hôpital. Ces décès étaient dus à la gravité des lésions du fait de la grande vélocité des projectiles, mais aussi aux longs délais d'évacuation des blessés 
vers l'hôpital. Cette mortalité élevée devraitt faire accentuer les mesures de protection individuelle et collective dans cette mission des Nations Unies au Mali.

\section{Conclusion}

Les traumatismes balistiques sont fréquents dans la mission des Nations Unies au Mali. Le respect des principes de la chirurgie de guerre conditionne le pronostic de ces traumatismes. Des mesures de protection individuelle et collective devraient être accentuées afin de limiter le risque d'exposition à ces lésions dans cette mission à fort défi sécuritaire. Les procédures d'évacuations des patients devraient être améliorées afin de réduire les délais d'évacuations.

\section{Conflit d'intérêt : aucun}

Remerciements : Nos remerciements à la hiérarchie militaire togolaise et au staff des Nations Unies pour les efforts consentis afin de faciliter les activités de l'HN2 Togo de Kidal.

\section{References:}

1. Bruner, D., Gustafson, CG., \& Visintainer, C. (2011). Ballistic injuries in the emergency department. Emerg Med Pract ; 13(12) :1-30.

2. Chauvin, F., Fischer, LP., Ferrandis, JJ., Chauvin, E., \& Gunepin, FX. (2002). L'évolution de la chirurgie des plaies de guerre des membres en 1914-1918. Histoires des Sciences Med ; 2 : 157-173.

3. Club des Anesthésistes-Réanimateurs et Urgentistes Militaires. (2008). Les traumatismes balistiques. Marseille: La Revue du CARUM - Réanoxyo 23.

4. Daban, JL., Peigne, V., Boddaert, G., Okoue-Ondo, R., Paul, S., \& Debien, B. (2012). Traumatisme pénétrant et balistique. In SFAR, Conférences d'actualisation. Congrès national d'anesthésie et de réanimation. Paris : Elsevier ; pp 101-16.

5. Donat, N., Pasquier, P., Clapson, P., Perez, JP., \& Debien, B. (2009). Epidémiologie des blessures de guerre. Urgences ; 653 -66.

6. Fabeck, L., Hock, N., Goffine, J., \& Ngatchou, W. (2017). Notions de balistique et prise en charge des plaies par balle au niveau des membres. Rev Med Brux ; 38 : 474-81.

7. Giannou, C., \& Baldan, M. (2009). War surgery. Working with limited resources in armed conflict and other situations of violence. Volume 1. Geneve : ICRC. 
8. Haouam, F., Tabet, N., \& Nebbal, M. (2018). Traumatismes balistiques crânio-cérébraux: Facteurs pronostiques. Rev Int des Services de Santé des Armées ; 91(4) : 31-35.

9. Haus-Cheymol, R., Bouguerra, C., Mayorg, E., Nivoix, P., Prat, N., Verret, C., Duron, S., Mayet, A., Meynard, JB., Pommier de Santi, V., Decam, C., Pons, F., \& Migliani, R. (2011). Blessures par armes à feu et engins explosifs dans les armées. Résultats de la surveillance épidémiologique de 2004 à 2008. Méd et armées ; 39(1) : 89-96.

10. Khechimi, M., \& Maalla, R. (2015). Particularités des traumatismes balistiques au niveau des membres - à propos de 12 cas. Chir de la Main ; 34(6) : 387.

11. Rouvier, B., Lenoir, B., \& Rigal, S. (1997). Les traumatismes balistiques. Conférence d'actualisation. Paris : Elsevier ;703-16

12. Saint-Julien, J., Auroy, Y., \& Pons, F. (2013). Spécificité de la chirurgie de guerre et capacités du service de santé des armées. Bull Acad Natle Méd ; 197(9) : 1713 - 26. 